\title{
Quantum Statistics of Random Walks
}

\author{
Manfred Harringer \\ Independent Researcher, Cologne, Germany \\ Email: manfred.harringer@t-online.de
}

How to cite this paper: Harringer, $M$. (2018) Quantum Statistics of Random Walks. Journal of Modern Physics, 9, 1448-1458. https://doi.org/10.4236/jmp.2018.97089

Received: May 16, 2018

Accepted: June 24, 2018

Published: June 27, 2018

Copyright (C) 2018 by author and Scientific Research Publishing Inc. This work is licensed under the Creative Commons Attribution International License (CC BY 4.0).

http://creativecommons.org/licenses/by/4.0/

\begin{abstract}
The paper dealt with quantum canonical ensembles by random walks, where state transitions are triggered by the connections between labels, not by elements, which are transferred. The balance conditions of such walks lead to emission rates of the labels. The labels with emission rates definitely lower than 1 are like modes. For labels with emission rates very close to 1 , the quantum numbers are concentrated around a mean value. As an application I consider the role of the zero label in a quantum gas in equilibrium.
\end{abstract}

\section{Keywords}

Random Walks, Particle Statistics, Boson Statistics, Balance Conditions, Detailed Balance, Quantum Gas, Perron-Frobenius Theory

\section{Introduction}

In [1] quantum statistics starts with the grand canonical ensemble. The quantum canonical ensemble is mentioned, but not elaborated. I want to fill this gap. There is a simple example, which corresponds to a quantum canonical ensemble: There are $K$ employers and $N$ employees. I want to describe the fluctuation of employees between the employers. I assume, that there are rates $\left(\alpha_{i j}\right)$ for the preference of a change from employer $i$ to employer $j$. I observe the numbers $q(i)$ of employees per employer $i$ during some years. Trying to explain the fluctuations, there are two different models available. If I assume, that always the employees decide to change, the numbers $q(i)$ will follow particle statistics, i.e. they are gaussian like concentrated around a mean value. If I assume, that always the employers decide (without considering anything about employees), the numbers will follow quantum statistics. Then the values of $q(i)$ along such a fluctuation process are similar to a mode ([2] p. 100): for a mode $i$ there is a value $r$, where $\left.p(q(i)=n)=(1-r) * r^{n}\right)$, excepted for the values of the employer, 
who is most preferred by the change rates $\left(\alpha_{i j}\right)$, where there are many options. Such a process is defined in Chapter 2, with the employees as elements, the employers as labels, and the preference rates as request probabilities.

Normally both models about reasons of fluctuations are mixed. But in statistical physics there is a clean cut. In [1] Huang introduces different kinds of elements: particles, bosons and fermions. Then particles are treated in particle statistics, bosons and fermions in quantum statistics. I search for such differences elsewhere, in the trigger method of state transitions. In my quantum systems it is possible, that a request for a transition is rejected, because there is no element available to perform the transition. In my example above it is artificial. But it is essential, when I choose such a model.

In [3] states and state changes are described by transition probabilities of complexes. I consider most simple complexes, i.e. single exchanges between species (labels). In [3] the number of particles per species (label) is observed along several steps of a transition process. There are balance conditions for the transition process (in a special case, [3] 16.3). Instead of transition probabilities for particles I build a quantum analogue by request probabilities (Chapter 2) with nearly the same balance conditions (Chapter 4). The common feature is a unique eigenvector (up to a factor $\lambda>0$ ), unique because of the theorem of Perron-Frobenius ([3], Chapter 16.6). In [3] the eigenvector consists of probabilities with sum $=1$. In the corresponding quantum system it consists of emission rates, where the highest emission rate has a value between $1 / K$ and 1 .

There is an important special case, (dynamic) equilibrium, i.e. detailed balance (Chapter 5). When there is given a positive vector (or function) $\rho$, there exist transition probabilities for particles for an exchange process in detailed balance ([4] Metropolis algorithm, or with $\rho(x)=\exp (-\beta H(x))$ in the hybrid Monte Carlo method). I use the same values as request probabilities. The eigenvector $\rho$ is the same for all numbers $N$ of elements of the exchange process. When $N=1$, transition probabilities and request probabilities coincide. When $N$ increases, the emission rates increase by a common factor. Another setup is: there is a space $X$, where I can build approximately equal distributed finite sets, with a function $H: X \rightarrow \mathbb{R}$. Then the selection of these finite sets varies, and one asks for properties of the exchange processes, which are independent of the selection. Assuming equilibrium, the determination of the edges of the state transitions is less important than it is e.g. in models of equilibration as in [5] for quantum systems, or in approximation tasks by the Metropolis-Hastings algorithm [4].

My main reference is [3] (which mentions many additional references) because of the balance conditions and the eigenvector. Then I search for suitable labels to count elements. The particle systems in [3] are not related to any mechanical particle motions. Therefore the labels must not be related to the moving objects of quantum mechanics, and it is not required, that single steps are unitary transformations as in usual quantum random walks [6]. Instead of ensembles ([4] Chapter 10.1) I observe the routes of random walks to derive 
probabilities and mean values. For connections to statistical physics I use [1] or [4] as main reference for an ideal quantum gas and the black box radiation. There I find suitable labels (Chapter 6).

I started to consider such random walks, trying to explain the difference between Boltzmann and Gibbs entropy more explicitly than in [7]. It was my "Gibbs version" of the random walks, which led to my version of quantum random walks. I made numerous computer simulations, observing the results of such random walks, to confirm my theoretical considerations.

\section{Random Walks}

Given a directed, connected graph $\Gamma(V, E)$ with $K$ vertices (labels) $V=\{1, \cdots, K\}$, and edges $e \in E$. An edge e leads from label $\operatorname{start}(e)$ to label end(e). For labels $i$, $j$ with $i \neq j$, there is at most one edge $e \in E$ with $i=\operatorname{start}(e)$ and $j=e n d(e)$. Then I write $e=(i \rightarrow j)$. The inverse edge is $(-e)=(j \rightarrow i)$. The graph is assumed to be homogeneous: When $e \in E$, then is $-e \in E$. There is a number $L$, that for all labels $i$

$$
\#\{e \in E \mid \operatorname{start}(e)=i\}=\#\{e \in E \mid \operatorname{end}(e)=i\}=L
$$

Given a function of request probabilities

$$
\alpha:\left\{\begin{array}{l}
E \rightarrow[0,1] \\
e \rightarrow \alpha_{e}
\end{array}\right.
$$

For $N>0$ I define random walks through

$$
Q:=\left\{q: V \rightarrow \mathbb{N}_{0} \mid \sum_{i=1}^{K} q(i)=N\right\}
$$

A single step of the random walk consists of a request and a transition:

request of an edge:

select an edge $e \in R$ randomly, with same probability for each edge select a random number $\tau \in[0,1)$

if $\left(\tau<\alpha_{e}\right)$, the request is accepted, otherwise rejected

transition, if the request of edge $e=(i \rightarrow j)$ is accepted at $q \in Q$ : (2.5)

if $(q(i)>0)$, the transition is accepted and performed by $q \rightarrow r$ with $\quad r(i)=q(i)-1 \quad$ (annihilation at vertex $i$ ) and $r(j)=q(j)+1 \quad($ creation at vertex $j), r(k)=q(k)$ at $k \neq i, j$ if $(q(i)=0)$, the transition is rejected

On rejection $\mathrm{q}$ is not changed.

I call it a quantum process. One version of a corresponding particle process is: I select a particle. It is at label $i$. Therefore I select an edge starting at $\mathrm{i}$ to perform the transition.

Each function $\alpha$ defines a $(K \mathrm{x} K)$-matrix $A=\left(\alpha_{i j}\right)$ by

$$
\alpha_{i j}:=\left\{\begin{array}{l}
\alpha_{e} \text { for } e=(i \rightarrow j) \text { and } e \in E \\
0 \text { if there is no } e=(i \rightarrow j) \in E
\end{array}\right.
$$




\section{Evaluations}

The result of a finite random walk is summarized by the number of all accepted requests, where $q(i)=n$ for the current state $q$ (i.e. where the transition will start):

$$
c(i, n):=\{\text { accepted requests } \mid q(i)=n\}
$$

The probability for a quantum number $n$ at a label $i$ is

$$
p(q(i)=n):=\frac{c(i, n)}{\sum_{m=0}^{N} c(i, m)}
$$

The mean quantum number at a label $(i)$ is

$$
\langle q(i)\rangle:=\frac{\sum_{n=0}^{N} n \cdot c(i, n)}{\sum_{n=0}^{N} c(i, n)}
$$

More typical for a quantum process is the emission rate:

$$
r(i):=p(q(i)>0)=\sum_{n=1}^{N} p(q(i)=n)
$$

i.e. the probability, that a transition starting at label $\mathrm{i}$ is accepted, related to all accepted requests starting at label $i$. The requests are independent of the current state. Therefore counting at all accepted requests leads to the same probabilities.

A special case is $N=1$. Then $r(i)=p(i)$, the probability, that the only element is at label $i$. Particle statistics of $N>1$ elements can be explained by $N$ identical and independent systems of such a 1-element system.

\section{Balance Conditions}

The probability of an accepted transition, which ends at a label $i$ (input for $i$ ), must be equal to the probability of an accepted transition, which starts at $i$ (output from $i$ ). Then, regarding (2.1)

$$
\sum_{(e \in E, \text { end }(e)=i)}\left(\alpha_{e} \cdot r(\operatorname{start}(e))\right)=\left(\sum_{(e \in E, \operatorname{start}(e)=i)}\left(\alpha_{e}\right)\right) \cdot r(i)
$$

Therefore the values $r(i)$ build an eigenvector of the matrix (2.6), supplied with diagonal elements as in [3]

$$
\alpha_{i i}:=-\left(\sum_{(e \in E, \operatorname{start}(e)=i)} \alpha_{e}\right)
$$

As suggested in [3], I can add a common value $\lambda>0$ to the diagonal elements, to achieve non negative matrix elements. The matrix must be irreducible. Then the values $r(i)$ build an exemplar of the unique eigenvector with positive components due to the theorem of Perron-Frobenius.

The balance condition (4.1) is a striking property of such systems. In [3] it is considered only for particle statistics.

Let $t(i):=$ (number of all accepted transitions starting at label $i$ ) $\approx$ (number of all accepted transitions ending at label $i$ ). I define

$$
p((i, n+1) \rightarrow(i, n)):=\frac{c((i, n+1) \rightarrow(i, n))}{t(i)}
$$




$$
p((i, n) \rightarrow(i, n+1)):=\frac{c((i, n) \rightarrow(i, n+1))}{t(i)}
$$

Then there is another balance condition, which is used in a similar context by Einstein to derive Planck's radiation law ([8], and there are many related presentations available).

For a label $i$ and a quantum number $\mathrm{n}$ there is

$$
p((i, n) \rightarrow(i, n+1))=p((i, n+1) \rightarrow(i, n))
$$

I search for a relation to modes in my context. The probability of an accepted request of edge $e$ in all accepted requests is

$$
p(\text { request of } e)=\frac{\alpha_{e}}{\sum_{(b \in E)} \alpha_{b}}
$$

The probability, that an accepted request starts at label $i$ with quantum number $\mathrm{n}$ related to all accepted requests, is

$$
p(i, n)=\frac{\sum_{(\operatorname{star}(e)=j)} \alpha_{e}}{\sum_{(b \in E)} \alpha_{b}}
$$

The probability of a rejection of a transition $i \rightarrow j$ depends on the actual quantum number at the end of the edge, i.e. $q(j)$, because a higher value of $q(j)$ gives a higher probability of the rejection condition " $q(\hat{i})=$ zero" at the start of the edge, label $i$. I have probabilities like " $p(q(\operatorname{start}(e))=m) \& q(\operatorname{end}(e))=n)$ ". If I assume independence, I get a relation:

$$
p(q(\operatorname{start}(e)=m) \& q(\operatorname{end}(e))=n)=p(q(\operatorname{start}(e)=m)) \cdot p(q(\text { end }(e))=n)
$$

Then I get via (3.4)

$$
\begin{gathered}
p((i, n) \rightarrow(i, n+1))=\frac{\sum_{(e n d(e)=i)} \alpha_{e} \cdot r(\operatorname{start}(e)) \cdot p(q(i)=n)}{\sum_{(b \in E)} \alpha_{b}} \\
p((i, n+1) \rightarrow(i, n))=\frac{\sum_{(\operatorname{start}(e)=i)} \alpha_{e} \cdot p(q(i)=n+1)}{\sum_{(b \in E)} \alpha_{b}}
\end{gathered}
$$

The balance (4.2) is

$$
\begin{gathered}
\sum_{(\text {end }(e)=i)} \alpha_{e} \cdot r(\operatorname{start}(e)) \cdot p(q(i)=n)=\sum_{(\operatorname{start}(e)=i)} \alpha_{e} \cdot p(q(i)=n+1) \\
\frac{p(q(i)=n+1)}{p(q(i)=n)}=\frac{\sum_{(e n d(e)=i)} \alpha_{e} \cdot r(\operatorname{start}(e))}{\sum_{(\operatorname{start}(e)=i)} \alpha_{e}}
\end{gathered}
$$

Therefore the quotient is independent of the quantum number $n$. Such a constant quotient, if $<1$, describes the distribution of the quantum numbers of a mode ([2] p. 100). Therefore I can try to compare the labels with modes. For a perfect mode at label $i$ there would be 


$$
\langle q(i)\rangle=\frac{r(i)}{1-r(i)}=\frac{1}{\frac{1}{r(i)}-1}
$$

In the counting results of my random walks (4.4) the values are nearly equal, excepted when the value of $r(i)$ is close to 1 .

\section{Equilibrium}

Given a vector

$$
\rho: V \rightarrow \mathbb{R}_{+}
$$

I define "equilibrium" by request probabilities for each edge $e=(i \rightarrow j) \in E$ by

$$
\alpha_{(i \rightarrow j)}:=\min \left\{1, \frac{\rho(j)}{\rho(i)}\right\}
$$

as usual in the Metropolis algorithm or via $\log \rho$ instead of $\rho$ in the Hybrid Monte Carlo algorithm [4] for transition probabilities. The vector $\rho$ fulfills the detailed balance conditions, because for each pair $((i \rightarrow j),(j \rightarrow i))$ of edges there is

$$
\alpha_{(i \rightarrow j)} \cdot \rho(i)=\min \{\rho(i), \rho(j)\}=\alpha_{(j \rightarrow i)} \cdot \rho(j)
$$

Therefore especially the balance conditions (4.1) are fulfilled by the vector $\rho$. The equilibrium for $\rho$ is independent of the set of edges $E$, which is used for the transitions. Only (5.2) and irreducibility of the matrix (2.6) is required (for (2.1) one adds edges with request probabilities 0 ). In the context of the Metropolis-Hastings algorithm [4] there are additional options to vary.

Such balance conditions are not available in the boson system of [1] Chapter 8.5, which consists of a set of independent labels $p$, because there the precondition ([1] (8.57) $\Sigma n_{p}=N$ ) is eliminated due to the passage to the grand partition function ([1] (8.61)). It leads to independent labels and balance conditions like (4.2) at each single label $p$. Along a random walk, the edge selection is independent of the current state. Therefore I can interpret the random walk through $Q$ as a random walk with $Q$ as set of labels of a single element $(K=\# Q, N=1)$, extending $\rho: V \rightarrow \mathbb{R}_{+}$to

$$
\rho:\left\{\begin{array}{l}
Q \rightarrow \mathbb{R}_{+} \\
q \rightarrow \prod_{i=1}^{K} \rho(i)^{q(i)}
\end{array}\right.
$$

The request probability of an edge $(q \rightarrow r)$ for $q, r \in Q$ is

$$
\alpha_{(q \rightarrow r)}:= \begin{cases}\alpha_{(i \rightarrow j)} & \begin{array}{l}
\text { when there is an edge }(i \rightarrow j) \in E \text { with } q=r, \\
\text { excepted } q(i)-1=r(i) \text { and } q(j)+1=r(j) \\
\text { otherwise }
\end{array}\end{cases}
$$

I can fulfill condition (2.1) e.g. adding multiple edges $(q \rightarrow q)$.

The extended vector $\rho$ fulfills the corresponding detailed balance conditions 
(5.3). As mentioned before, because of $N=1$, there is

$$
r(q)=p(q)=\text { probability of occurrences of } q \text { in the random walk }
$$

Now $N$ may vary. I write $Q(N)$ for $Q$ defined by (2.3), $p_{N}$ instead of $p$ and $r_{N}$ instead of $r$. Then with $Z_{0}:=1$ and

$$
\begin{aligned}
& Z_{N}:=\sum_{(q \in Q(N))} \rho(q)=\text { the complete homogeneous symmetric polynomial } \\
& \text { in } K \text { variables } \rho(i) \text { of degree } N \\
& p_{N}(q)=\frac{\rho(q)}{Z_{N}}(\text { probabilities have sum }=1) \\
& p_{N}(q(i)=n)=\sum_{(q \in Q(N), q(i)=n)} \frac{\rho(q)}{Z_{N}} \\
& r_{N}(i)=p_{N}(q(i)>0) \\
& =\sum_{(q \in Q(N), q(i)>0)} p(q) \\
& =\sum_{(q \in Q(N), q(i)>0)} \frac{\rho(q)}{Z_{N}} \\
& =\rho(i) \cdot \frac{Z_{N-1}}{Z_{N}}(\operatorname{using}(5.4)) \\
& \frac{r_{N}(i)}{r_{1}(i)}=Z_{1} \cdot \frac{Z_{N-1}}{Z_{N}}(\text { independent of } i)
\end{aligned}
$$

It confirms, that for each $N$ the emission rates are multiples of the same eigenvector, and that $N=1$ leads to probabilities. Furthermore $r_{N}(i)$ increases with $N$ and the limit for

$N \rightarrow \infty$ is 1 (proof via Z-functions). Because of (5.6) the emission rates (more general: all probabilities of quantum numbers) are independent of variations of the edges and request probabilities as mentioned at (5.3), detailed balance. Writing $\rho(i)=\exp (-\beta H(i))$ I get Z-functions as usual.

\section{Applications}

\subsection{Main Results}

Normally the labels are like modes, but there are exceptions. If $\rho(i)$ is maximal at label $i$, there are two significant types. The label may be like a mode too (typically at low values of $\beta$ or low values of $N$ ), or its quantum numbers may be concentrated, i.e. nearly gaussian distributed around a mean value (typically at large values of $\beta$ or large values of $N$ ). There are continuous passages between these types.

Table 1 contains counting results and derived results for $K=12$ labels with values $H(i)$, randomly distributed in $[0,1]$, labels sorted by the $H$-values, $\beta=1$, $N=80$. The random walk consists of $10^{10}$ steps. Evaluations start at $1 / 2$ of all steps, to achieve a randomized start position.

Now I look at special functions $\rho$. In the context of quantum gases I find a 
Table 1. Emission rates and mean values for random values of $H(i)$.

\begin{tabular}{|c|c|c|c|c|c|c|}
\hline label i & $H(i)$ & $r(i)$ & $t(i)$ & $\langle q(i)\rangle$ & $\langle r-\operatorname{mode}(i)\rangle$ & $\langle t-\operatorname{mode}(i)\rangle$ \\
\hline 1 & 0.042093 & 0.997428 & 0.997384 & 43.576472 & 387.742031 & 381.324757 \\
\hline 2 & 0.117060 & 0.925058 & 0.925348 & 11.658032 & 12.343617 & 12.395400 \\
\hline 3 & 0.121573 & 0.920967 & 0.921181 & 11.109490 & 11.652974 & 11.687266 \\
\hline 4 & 0.207110 & 0.845804 & 0.845661 & 5.426121 & 5.485244 & 5.479257 \\
\hline 5 & 0.506089 & 0.626902 & 0.627121 & 1.678370 & 1.680264 & 1.681837 \\
\hline 6 & 0.694566 & 0.519745 & 0.519394 & 1.080761 & 1.082228 & 1.080706 \\
\hline 7 & 0.706656 & 0.512974 & 0.513153 & 1.054312 & 1.053278 & 1.054032 \\
\hline 8 & 0.754032 & 0.489525 & 0.489408 & 0.958108 & 0.958959 & 0.958512 \\
\hline 9 & 0.766346 & 0.483657 & 0.483419 & 0.936427 & 0.936696 & 0.935804 \\
\hline 10 & 0.813673 & 0.461302 & 0.461073 & 0.855559 & 0.856327 & 0.855538 \\
\hline 11 & 0.819835 & 0.458266 & 0.458241 & 0.845412 & 0.845926 & 0.845838 \\
\hline 12 & 0.836762 & 0.450872 & 0.450549 & 0.820937 & 0.821070 & 0.819999 \\
\hline
\end{tabular}

$r(i)=$ emission rate of label $i$ by the counting results $c(i, n) ; t(i)=$ emission rate of label $i$ by Z-functions (5.6) ("theoretical"); $\langle q(i)\rangle=$ mean quantum number at label $i$ by the counting results $c(i, n)$; $\langle r$-mode $(i)\rangle=$ $r(i) /(1-r(i))=$ mean quantum number of the corresponding mode $\langle t$-mode $(i)\rangle=t(i) /(1-t(i))$.

basic relation of Bose statistics in [9] (11.4):

$$
\left\langle n_{k}\right\rangle=\frac{1}{\exp \left(\beta\left(\varepsilon_{k}-\mu\right)\right)-1}
$$

It means: A vector as required in (5.1) is

$$
\rho(k)=\exp \left(-\beta \varepsilon_{k}\right)
$$

I assume Equilibrium (5.2) of a quantum process. Then $\rho(k)$ becomes an eigenvector, because of the detailed balance (5.3). The emission rates build an eigenvector too. I assume, that there is a unique maximum of $\rho$ in $k=1$ (i.e. $\varepsilon_{1}$ is minimal). Then there is a unique $\mu<\varepsilon_{1}$ with $r(1):=\exp \left(-\beta\left(\varepsilon_{1}-\mu\right)\right)$, and therefore for each label $k$

$$
r(k)=\exp \left(-\beta\left(\varepsilon_{k}-\mu\right)\right)
$$

Then (6.1) coincides with (4.4), which is well approximated in my random walks for labels $k>1$. But the label $k=1$ is an exception, not for (6.2), but for (6.1). Therefore $k=1$ is treated by an extra term in [1] and [4].

\subsection{Ideal Bose gas}

If I assume detailed balance of the exchange of kinetic energy in an ideal Bose gas [1] or [4], I have 3D vectors $p$ with

$$
\begin{gathered}
\varepsilon_{p}=\frac{|p|^{2}}{2 m} \\
\rho_{\beta}(p)=\exp \left(-\beta \cdot \frac{|p|^{2}}{2 m}\right)
\end{gathered}
$$


In such systems there exist critical temperatures, where the label $(p=0)$ is no longer like a mode (Bose-Einstein condensation). One finds a critical temperature on this way [10]: I build families of systems by their Hamilton operator ([10] (1.4)). $\beta$ is fixed. I calculate the value of the emission rate $r(p=0)$ (called fugacity in this context) for single members of the family. Then I build a limit of the systems, running to infinity (there it is $L \rightarrow \infty)$. Although $r(p=0)<1$ for each single system, the limit may be 1 . Then $\beta$ varies, and the critical value $\beta_{c}=1 / k T_{c}$ is the minimal $\beta$ (maximal temperature), where the limit is 1 . Then I can show, that in the limit the zero label $(p=0)$ has too many elements in mean, to be like a mode. It remains valid to claim, that for finite systems there are continuous passages between the different types of behavior at the label $(p=0)$.

The effect of Bose-Einstein condensation is observed in [11] based upon a birth and death process. It contains steps modeled by single transitions from one species to another. I guess, that there exist steps with rejections because of an empty resource. But I cannot find it.

\subsection{Photon Gas}

If I assume detailed balance of the exchange of the photon energy in an ideal photon gas ([1] Chapter 12.1), I have

$$
\rho_{\beta}(k)=\exp \left(-\beta \cdot \hbar \omega_{k}\right)
$$

with $\omega_{k}:=c|k|$ and Planck's constant $\hbar$.

The label $k$ is a $3 \mathrm{D}$-vector. The setup for the random walks is similar as in (6.2). But here instead of (6.1) there is in [1] (12.8)

$$
\left\langle n_{k}\right\rangle=\frac{2}{\exp \left(\beta \cdot \hbar \omega_{k}\right)-1}
$$

"2" indicates, that there are two labels for each vector $k$, because of two different polarizations. There are several options to determine the labels: When there are two labels, I am asked about the request probabilities for transitions between these labels (which are $=1$, because here the $\omega_{k}$-values are equal). Or I assume one common label for both polarizations, because the exchange is independent of the polarization. Or there is no exchange between different polarizations and therefore I have two independent systems of the same kind.

Apart from the factor 2 it is just black box radiation. The idea behind (6.5) is, that in this case all labels are like modes. But there exist experimental results about special photon gases, where there is an exceptional zero label [12]. In [13] it is explained assuming a grand canonical ensemble in the case of modes and a canonical ensemble in the exceptional case.

Table 2 contains counting results and derived results for a photon gas model, which includes both cases. The setup is: $H(x)=|x|$, a 3D ball of radius 6 with $K=$ 2000 labels equal distributed in the ball, 0 is included, $\beta=0.9$. It leads to maximal energies per value $|x|$ at $|x| \approx 3.0 \ldots 3.3$. Each random walk consists of $10^{10}$ steps. Evaluations start at $1 / 2$ of all steps. The number of elements $\mathrm{N}$ is varied. 
Table 2. Emission rates and mean values for a finite model of a photon gas.

\begin{tabular}{cccccccc}
\hline $\mathrm{N}$ & $\boldsymbol{r}(0)$ & $\boldsymbol{r}(\boldsymbol{y})$ & $\langle q(0)\rangle$ & $\langle q(y)\rangle$ & $\langle\operatorname{mode}(0)\rangle$ & $\langle\operatorname{mode}(y)\rangle$ & zeroes \\
\hline 60 & 0.713194 & 0.507569 & 2.288819 & 1.024469 & 2.486677 & 1.030741 & 0 \\
80 & 0.901743 & 0.628189 & 6.458483 & 1.652551 & 9.177413 & 1.689536 & 0 \\
100 & 0.978373 & 0.694037 & 14.391452 & 2.237323 & 45.238498 & 2.268371 & 0 \\
120 & 0.998734 & 0.709060 & 31.147484 & 2.430359 & 789.014220 & 2.437137 & 0 \\
140 & 1.000000 & 0.707658 & 54.831132 & 2.394297 & none & 2.420653 & 9 \\
160 & 1.000000 & 0.705996 & 73.949548 & 2.400769 & none & 2.401319 & 32 \\
180 & 1.000000 & 0.705799 & 92.366046 & 2.385630 & none & 2.399034 & 46 \\
\hline
\end{tabular}

" 0 " is the zero label; label $y$ is the nearest neighbor of the zero label; $r(i)=$ emission rate of label $i$ by the counting results $c(i, n) ;\langle q(i)\rangle=$ mean quantum number at label $i$ by the counting results $c(i, n)$; $\langle$ mode $(i)\rangle$ $=r(i) /(1-r(i))=$ mean quantum number of the corresponding mode; zeroes $=\max \{n \mid c(0, v)=0$ for each $v$ $<n$.

At $N=60$ label 0 is like a mode. Then there is a continuous passage, and beginning at $N=120$, label 0 becomes concentrated around a mean value and $r(y)$ no longer increases. Although the random walks are rather long, the values have random deviations, because $K$ is large. As a counting result, I get $r(0)=1$. The theoretical value remains $<1$. The values of the corresponding Z-functions in (5.6) are around $10^{31}$.

\section{Conclusions}

I considered random walks, where state transitions are triggered by the connections between labels, not by elements, which are transferred. The balance conditions of such walks lead to emission rates of the labels. The labels cannot be exactly like modes, in contrast to [2] (12.2). They are not connected with electromagnetic waves and their superposition principle. But I can build models of dynamical equilibrium, which gain insight into the statistics of the quantum numbers, and I can try to compare it e.g. with a Bose-Einstein condensate. There is no singularity, because the zero label is not introduced as a mode with mean quantum number, but as a label with an emission rate. To get comparable quantitative results there is an important question left open: How are the numbers of elements and labels related to pressure and volume of a quantum gas?

Another question is: Are there systems, where the zero label of my finite quantum system can explain the properties of the vacuum of a corresponding quantum system?

Comparisons are difficult, because I considered equilibrium only. It is left open, how to introduce a quantitative notion of time.

\section{References}

[1] Huang, K. (1987) Statistical Mechanics. 2nd Edition, John Wiley \& Sons, New York.

[2] Kittel, C. and Kroemer, H. (1989) Physik der Wärme. 3rd Edition, R. Oldenbourg, 
Munic, Oldenburg.

[3] Baez, J.C. and Biamonte, J.D. (2012) A Course on Quantum Techniques for Stochastic Mechanics. arXiv:1209.3632v1 [quant-ph].

[4] Tuckerman, M.E. (2010) Statistical Mechanics: Theory and Molecular Simulation. Oxford University Press, Oxford.

[5] Wolschin, G. (2018) Physica A, 499, 1-10. https://doi.org/10.1016/j.physa.2018.01.035

[6] Kempe, J. (2003) Quantum Random Walks-An Introductory Overview. arXiv:quant-ph/0303081v1.

[7] Miranda, E.N. (2015) Journal of Modern Physics, 6, 1051-1057. https://doi.org/10.4236/jmp.2015.68109

[8] Einstein, A. (1917) On the Quantum Theory of Radiation. Physikalische Zeitschrift Bd. 18.

[9] Plischke, M. and Bergerson, B. (2005) Equilibrium Statistical Physics. 3rd Edition, World Scientific Publishing, Hackensack.

[10] Davies, E.B. (1972) Communications in Mathematical Physics, 28, 69-86. https://doi.org/10.1007/BF02099372

[11] Bianconi, G., Ferretti, L. and Franz, S. (2009) Non-Neutral Theory of Biodiversity. Europhysics Letters, 87, P07028. https://doi.org/10.1209/0295-5075/87/28001

[12] Damm, T., Schmitt, J., Qi Liang, D. Dung, F. Vewinger, M. Weitz and J. Klaers, (2016) Nature Communications, 7, Article Number: 11340. https://doi.org/10.1038/ncomms11340

[13] Klaers, J., Schmitt, J., Vewinger, F. and Weitz, M. (2010) Nature, 468, 545-548. 